\title{
A NEW APPROACH OF EDGE DETECTION IN SAR IMAGES USING REGION-BASED ACTIVE CONTOURS
}

\author{
Ch.SriVeeraSagar', K.RamaDevi ${ }^{2}$ \\ ${ }^{1,2}$ Department of Electronics and Communications Engineering, JNTUK Kakinada, sairamsagar4472@gmail.com
}

\begin{abstract}
This paper presents a new methodology for the edge detection of complex radar images. The approach includes the edge improvisation algorithm and followed with edge detection. The nature of complex radar images made edge enhancement part before the edge detection as the data is highly heterogeneous in nature. Thus, the use of discrete wavelet transform in the edge improvisation algorithm is justified. Then region based active contour model is used as edge detection algorithm. The paper proposes the distribution fitting energy with a level set function and neighborhood means and variances as variables. The performance is tested by applying it on different images and the results are been analyzed.
\end{abstract}

Keywords: Edge detection, Edge improvisation, Synthetic Aperture radar (SAR), wavelet transforms.

$* * *$

\section{INTRODUCTION}

Image processing is a rapidly emergent area of computer science. Its growth has been uplifted by technological achievements in digital imaging, computer processors and mass storage devices. The flexibility and affordability of various streams are now made path to digital systems. Examples are medicine, remote sensing and security monitoring etc.., and the other sources also produce huge amount of digital image data day to day, which cannot ever be examined manually. Digital image processing is concerned primarily to extract useful information from images. Ideally, this is done by computers, with little or no human intervention. Image processing algorithms may be placed at three levels. At the lowest level are those techniques which deal directly with the raw, possibly noisy pixel values, with denoising and edge detection being good examples. In the middle are algorithms which utilize low level results for further means, such as segmentation and edge linking. At the highest level are those methods which attempt to extract semantic meaning from the information provided by the lower levels.

Synthetic aperture radar (SAR) has great ease of observation during the day as well night. This property made the SAR more superior than the optical space borne sensors and any other, as it has continuous observation over earth which is independent of sun light. Apart from the merits, the visual perception of SAR data for an inexperienced viewer is very difficult and cannot distinguish the meaningful data. So, the image processing techniques are used for the extraction of useful data. The available general methods cannot give the better image result compared to optical space borne sensor images. Hence, some particular algorithms are still to make use for the analysis of complex radar data. Novel edge detection techniques are essentially in two parts edge improvisation and detection. An edge enhancement phase is critical in providing intensive detection rates in multiscale images. There are many methods used for denoising normally such as Sobel filter [1], Prewitt filter [2], morphological operators etc.., but they are not up to the mark in synthetic aperture image applications. This is due to the presence of speckle noise, termed as multiplicative noise like pattern [3] in SAR images which made this phase critical. The paper gives the new approach for the edge enrichment of SAR images by the application of discrete wavelet transforms for the increased efficiency then the existing conventional methods to extract the information.

There are different models for the edge detection which can are having easy in application and low computational cost. As they are not quit efficient in the case of SAR images as it is having discontinuities of edges. The robust method proposed in this paper is base on region based active contour model. This algorithm using region based model to edge based model as it has better performance in the presence of noise and weak edge. Finally the successive use of the both proposed algorithms will tackle the subpixel level efficiency and robust even in the presence of multiplicative noise pattern in the images.

\section{REVIEW OF SAR IMAGES}

The design of SAR and its associated applications are growing exponentially since 1950's from the day Carl Wiley of the "Good year Aircraft Corporation", observed that a one to one communication exists between track coordinates of a reflecting object and the instantaneous Doppler swing of the signal to the radar by the object. By his conclusion it made the spurt path to performance of the real aperture radar (RAR) design.

In 1990's the significant expansion of SAR missions with the launch of five earth oriented SAR satellites along with other two more Shuttle Imaging Radar missions and other are still in use and in progress for improving the space 
observation. The new sensors have improved resolution in all dimensions, spatial resolution with reduced pixel sizes, sequential resolution with shorter repeat times and spectral resolution with increased number of spectral bands. By these new specifications, new challenges have appeared. The colossal amount of remote sensing data raises new computational issues. These applications are accessible or can achieve new results like change detection, natural disaster monitoring, landscape planning, biomass measurement detection of oil slicks. These advances are especially true for Synthetic Aperture Radar (SAR) sensors, with high resolution capablility.SAR images are difficult to interpret and new difficulties arose with the increase of spatial resolution.

The representation of SAR image can be explained on the basics convolution of the complex reflectivity of area $\alpha(\tau, \eta)$ with the impulse response of the SAR system $\mu_{0}(\tau, \eta)$ [3].

$$
\mu(\tau, \eta)=\alpha(\tau, \eta)^{*} \mu_{0}(\tau, \eta)
$$

Where $\tau$ and $\eta$ are the azimuth and the space coordinates, respectively, in use to define the coordinate system of a SAR image. The SAR system impulse response $\mu_{0}(\tau, \eta)$, the SAR data pixels are the low pass-filtered [4] version of the complex local scattering properties may be interpreted as a bidimensional low pass filter, in such a way the finite local support of this filter determines the spatial resolution of the SAR image. The SAR imaging process indicated by (1) can simplify by a mathematical model that considers linearity and Born approximation [5].

$$
\mu(\tau, \eta)=\sum_{\mathrm{k}=1}^{\mathrm{N}} \alpha_{\mathrm{k}}(\tau, \eta) * \mu_{\mathrm{o}}(\tau, \eta)
$$

Where $\alpha_{k}(\tau, \eta)$ is the complex reflectivity of the $\mathrm{k}^{\text {th }}$ individual scattering within the resolution cell. As speckle represent one of the major defaults for the access of useful information form SAR images. This can be filtered before the actual processing of the SAR images but with the conventional methods the speckle produces over segmentation even though they are able to smooth speckle. This leads to the unnecessary date present in the processed images as this results will be having poor spatially resolution. There are different speckle filters which are proposed in different literature, the commonly used are Lee filter [6], the gamma filter [7] and the frost filter [8]. In this paper we will be using one among the above mentioned filters along with the Sobel operator.

\section{GENERALISED EDGE IMPROVISATION ALGORITHM}

As we seen that the SAR image has multiscale nature, the combination of $\mathrm{N}$ individual scatter produces the speckle noise within the resolution cell which can be considered as the pixel level (i.e., which is significantly a small scale) spatial varying characteristic. For the justification of multiscale approach, the product model [9] formalizes a multiresolution notation stating intensity I as

$$
\mathrm{I}=\mu_{\mathrm{I}} \mathrm{Xn}
$$

Where $\mu_{\mathrm{I}}$ is mean intensity, $\mathrm{x}$ is the texture random variable and $\mathrm{n}$ is the speckle is also a random variable. $\mathrm{x}$ and $\mathrm{n}$ are considered statistically self-determining. Thus, (3) enables the identification of different ranges of scales, so in order to analyze SAR this approach is most reliable. As the wavelets transforms allow the time-frequency localization. It can also be used as the multiscale differential operator [10]. The Algorithm for edge improvisation in SAR images proposed in this paper relies on the difference of behavior along the wavelet scales of the speckle in face of the edges. The discontinuities are highlighted by the wavelet transform and they tend to persist over scales. Simultaneously the speckle is progressively smoothening; it is almost spatially uncorrelated between scales (see Fig. 1).

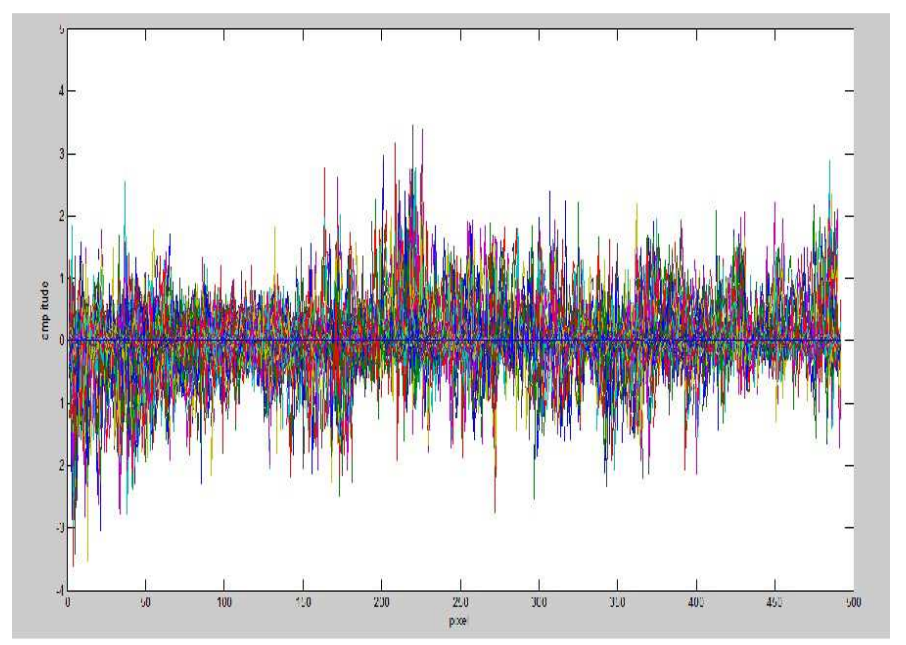

Fig-1. Simulated speckle signal is obtained through the application of Haar mother wavelet.

\section{A. ALGORITHM OF GENERALIZED EDGE IMPROVISATION}

The algorithm is proposed for various images ie., either one dimensional or two dimensional. The size of the window is also not concern for the application of this algorithm.

Step1: The image is first initialized, which has to be enhanced the distributions of scales are observed over the color bar. Some of the images are not smooth and does not monotonically progresses from dark to light. This type of indexed image is not suitable for direct wavelet decomposition with the toolbox and needs to be preprocessed. To convert the RGB images into a gray-scale intensity image, using the standard perceptual weightings functions for the three-color components. Then, convert the gray-scale intensity image back to a gray-scale indexed image with desired distinct levels and create a new colormap.

Step2: In SAR processing the application of logarithmic operation is used for the minimization of dynamic range of multiplicative speckle. The multiplicative noise transformed in this method is additive and signal independent, but its 
probability density distribution is approximately taken as Gaussian [11].

Step3: Generally the Haar transform is the oldest and simplest of all the wavelet transforms. Owing to its low computing capability, the Haar transform has been mainly used for pattern recognition and image processing. Hence, two dimensional signal and image processing is an area of efficient applications of Haar transforms due to their wavelet-like structure. Moreover, wavelets are considered as a generalization of the Haar functions and transforms. Such a transform is also well suited in communication technology for data processing, multiplexing and digital filtering.

The wavelet transform can be expressed as

$$
W(a, b)=\int_{a}^{b} f(x) \frac{1}{\sqrt{a}} \psi^{*}\left(\frac{x-b}{a}\right) d x
$$

The Haar wavelet is defined as

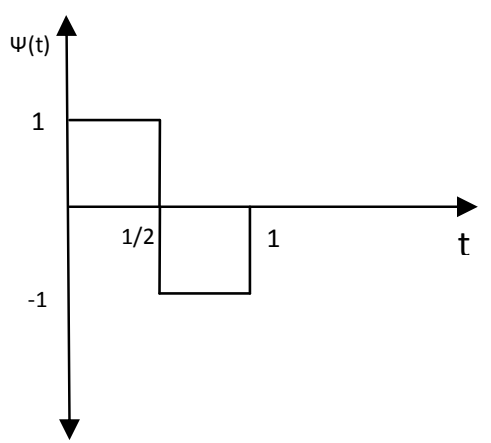

Fig-2. The graphical representation of Haar wavelet.

Hence, in the wavelet transform of the logarithm of the SAR signal, the influence of the speckle is low since its contribution in each of the semi intervals counteracts with the other, if we assume that the speckle is homogeneous through the observed scene. ie., the speckle in the larger intervals that obtained through the application of logarithm by the assumption of homogeneity made its values in that range very low and it can be taken as approximately zero.

Step4: Let us consider the subbands obtained through the application of wavelet transform: 1D wavelet transform has two frequency bands and the 2D wavelet transform has four bands for each level of decomposition. In 2D transform one band has the low pass filtered image and the rest will be in transformed in three orientations as horizontal, vertical and diagonal respectively. As the wavelets maximizes the discontinuities in the edges so, by taking the absolute value of the subbands (i.e., H,V\&D) the maximum values of the each band can be enhanced by applying normalization to the maximum.

Step5: Than by considering the pixel level local maxima in the each subband in the neighborhood of the discontinuity and multiplying these smallest resolution cells the discontinuities can be minimized and the speckle in the resolution cell will un-correlated which is divergent to the intrascale combination with the useful data and the edges are enhanced in each direction. This process is carried out for the different levels of scales.

The algorithm is preformed for the predefined number of iteration as the higher iterations of the algorithm may produce the thick edges. Compared to the other conventional methods such as Sobel gradient method the results shown in the figure (3) have reviled the good and much efficient method for the edge improvisation by the application of proposed method.

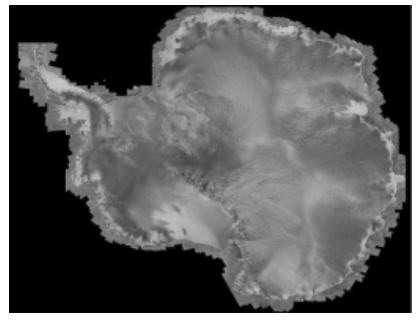

(a)

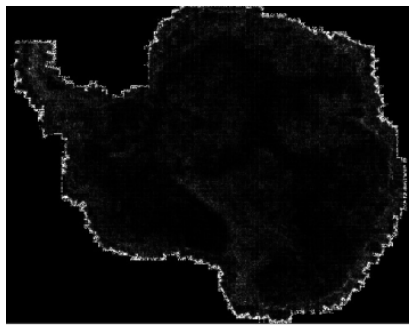

(c)

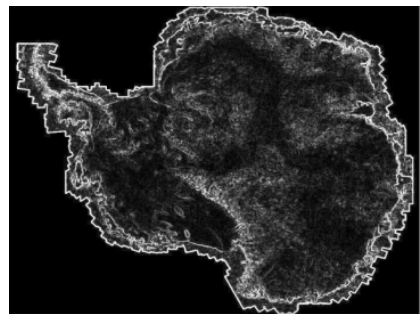

(b)

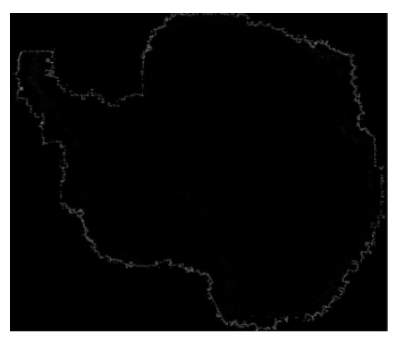

(d)
Fig-3. The result of the simulated images (a) it is the original image. (b) Result of Sobel gradient method. (c), (d) the result of proposed algorithm in second and third iteration. (Image is taken from Google SAR images courtesy).

As iteration count increases the edge will be much thinner than they appear, so the better implementation of the method for various images may be differ from one to another. The discussion on results can be elaborated in the result section.

\section{EDGE DETECTION WITH REGION-BASED MODELS}

Image segmentation is the one of the essential part in the scenario of image processing. There is been a great study especially in this class of area. An entrenched class of methods is active contour models, which have been widely used in image segmentation with promising results. The models can achieve subpixel accuracy and provide smooth contours. Active contour models can be mostly categorized into two models: edge-based models [12] and region-based models [13].Edge-based models uses image gradient to direct curve evolution, which are generally sensitive to noise and weak edges [14]. Instead of utilizing image gradient, region-based models usually intend to identify every region of interest by using a convinced region descriptor, such as intensity, texture or motion, to guide contour [15]. Therefore 
region-based models generally have better performance in the presence of image noise and weak object boundaries. In practice, intensity inhomogeneities occur in many real world images acquired with different modalities [16]. Especially, it is frequently seen in medical images and multi-resolution images.

Once the improvisation algorithm estimation has been obtained, a final step of edge detection is required to build an unsupervised algorithm. In our model, the image intensities are described by standard normal distribution with diverse means and variances. The paper proposes the distribution fitting energy with a level set function and neighborhood means and variances as variables. The energy minimization is achieved by an interleaved level set evolution and estimation of intensity means and variances in an iterative process. The means and variances of neighboring intensities are considered as spatially varying functions to handle noise of spatially varying strength (i.e., multiplicative noise). Then the new approach to the SAR images with multiplicative noise like pattern gives comparatively better results than the geodesic active contours which are driven by the edge based model [17].

Chan and Vese [18] developed a mean curvature flow based level set implementation of a specific case of the Mumford Shah energy functional, where the mean intensity of the pixels inside and outside the curve are used as a smooth approximation of the image but they are not optimum in the case of non homogeneous intensity images. So, by considering the LBF model proposed by C.Li [19] which is approximate the intensities inside and outside the contour. The LBF model cannot provide the required information for the perfect segmentation in the presence of heavy noise and intensity. So, by taking the input image from the above proposed algorithm speckle noise and the intensity inhomogeneities can be avoid as possible as maximum and the intensity can be taken from the normal probability distribution.

The energy function can be written as

$$
\begin{aligned}
& E_{f}\left(\phi, c_{1}, c_{2}\right)=\lambda_{1} \iint \mathrm{K}_{\sigma}(x-y) p_{1, x} I(y) H(\phi(y)) d y d x \\
& \quad+\lambda_{2} \iint K_{\sigma}(x-y) p_{2, x} I(y)(1-H(\phi(y))) d y d x
\end{aligned}
$$

Where $\lambda_{1}, \lambda_{2}, v, \mu$ are weighting positive constants. $K_{\sigma}$ is kernel function with a localization property that $\mathrm{K}_{\sigma}(\mathrm{x}-\mathrm{y})$ and the $\mathrm{C}_{1}, \mathrm{C} 2$ are the two regions in the image domain $\mathrm{C}$, the local point $\mathrm{x}$ with a curvature of $\mathrm{r}$. The kernel function is considered with the fixed $\sigma$. The two regions are considered as they are having mean and variance are spatially varying and defined as $u_{i}, \sigma_{i}{ }^{2}$ within the regions concern to local of $\mathrm{x}$. As the smoothing functions are defined in the Vese and Chan model [20] and C.Li [21] as

$L(\phi)=\mathrm{v} \int|\nabla \mathrm{H}(\phi(\mathrm{x}))| \mathrm{dx}$

Where $\phi$ is level set function, the $\mathrm{L}(\phi)$ is penalizing the length and for smooth contour propagation. The regularized level set evolution is from a distance function [22] which is for the accurate execution is given as

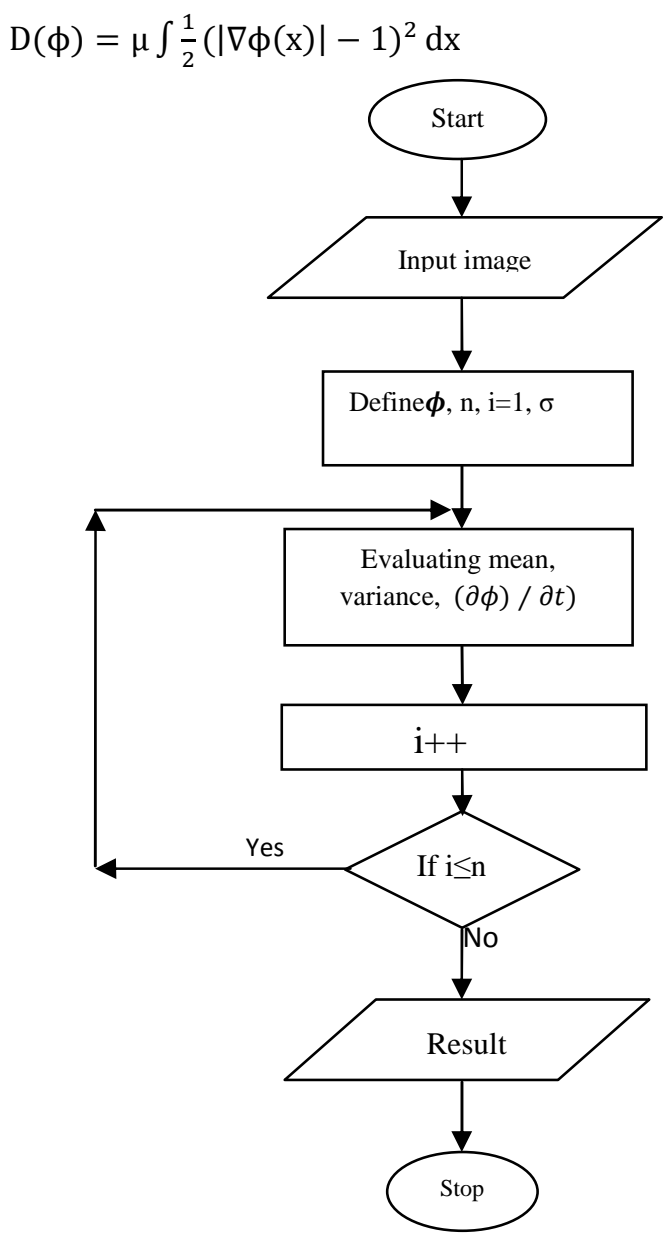

Fig-4. Flow chart for edge detection algorithm.

The energy function is considered after including the both smoothing and distance functions is

$\mathrm{F}\left(\phi, \mathrm{c}_{1}, \mathrm{c}_{2}\right)=\mathrm{E}_{\mathrm{f}}\left(\phi, \mathrm{c}_{1}, \mathrm{c}_{2}\right)+\mathrm{L}(\phi)+\mathrm{D}(\phi)$

This model is different from the C.Li and Chan Vese as we have taken the different distribution and also the application of method for specified images. The method is most complimentary with the iterative process done in various steps shown in the flow chart as follows.

\section{RESULTS}

The application of algorithms is absolutely gives the expected results for the costal line detection of SAR images as shown in figure. $5(\mathrm{a}) \&(\mathrm{~b})$. The simulation is performed in desktop of $2.8 \mathrm{GHz}$ processor with $2 \mathrm{~GB}$ ram in MatLab 2009 software. 


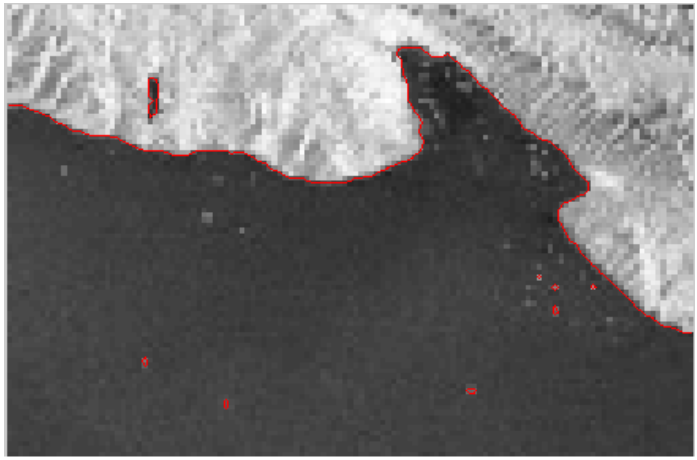

Fig-5. (a) the simulated image of detected edges by the application of algorithm proposed for edge detection of SAR images.

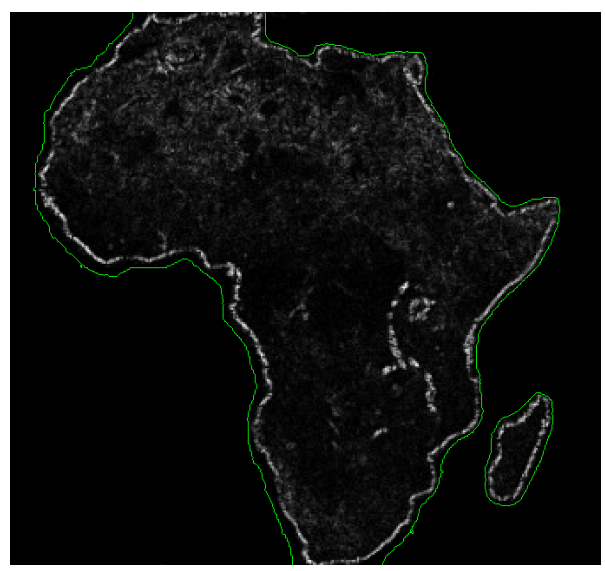

Fig-5. (b) the simulated image of extracted \& detected edges by the application of both algorithms proposed.

\section{CONCLUSION}

This paper is mainly focus on one of the problem general occurs in the SAR date and the detection of costal area boundaries of images for the application of remote sensing. The results are also good compared with other methods such as Sobel gradient method and geodesic active contours. As "snakes" are not so significant in detecting edges of most discontinuously formed with the application of wavelet transforms. So, the region based approach is most eligible method for the detection of edges in multiplicative noised images. The work can be further extended on 3D images and also for target and remote sensing applications with more reliability.

\section{REFERENCES}

[1] J. W. Goodman, "Speckle Phenomena in Optics: Theory and Applications," Englewood, CO: Roberts \& Company, 2007.

[2] V. Caselles, R. Kimmel, and G. Sapiro, "Geodesic active contours," Int. J.Comput. Vis., vol. 22, no. 1, pp. 61-79, Feb. 1997.
[3] C. Oliver and S. Quegan, "Understanding Synthetic Aperture Radar Images”. Norwood, MA: Artech House, 1998.

[4] J. C. Curlander and R. N. McDonough, "Synthetic Aperture Radar": Systems and Signal Processing. Hoboken, NJ: Wiley, 1991

[5] K. J. Langenberg, M. Brandfass, K. Mayer, T. Kreutter, A. Brüll, P. Felinger, and D. Huo, "Principles of microwave imaging and inverse scattering," EARSeL Adv. Remote Sens., vol. 2, no. 1, pp. 163-186, Jan. 1993.

[6] J. S. Lee, "Refined filtering of image noise using local statistics,Comput.Vis" Graph., Image Process., vol. 15, no. 4, pp. 380-389, Apr. 1981.

[7] A. N. Evans, "A gamma filter for multi-look synthetic aperture radar images," in Proc. ISSPA, 1996, pp. 829 832.

[8] R. Touzi, "A review of speckle filtering in the context of estimation theory," IEEE Trans. Geosci. Remote Sens., vol. 40, no. 11, pp. 2392-2404, Nov. 2002.

[9] F. T. Ulaby and C. Elachi, "Radar Polarimetry for Geoscience Applications", Norwood Artech House, 1990.

[10] S. Mallat, “A Wavelet Tour of Signal Processing”, New York: Academic, 1999.

[11] H. Arsenault and G. April, "Properties of speckle integrated with a finite aperture and logarithmically transformed," J. Opt. Soc. Amer., vol. 66, no. 11, pp. 1160-1163, Nov. 1976.

[12] M. Kass, A. Witkin, D. Terzopoulos, Snakes: active contour models, Int. J. Comput. Vis. 1 (4) (1987) 321331.

[13] R. Ronfard, Region-based strategies for active contour models, Int. J. Comput. Vis. 13 (2) (1994) 229-251.

[14] T. Chan, L. Vese, Active contours without edges, IEEE Trans. Image Process. 10 (2) (2001) 266-277.

[15] D. Cremers, M. Rousson, R. Deriche, A review of statistical approaches to level set segmentation: integrating color, texture, motion and shape, Int. J. Comput. Vis.Vol.72, no.2, (2007)195-215.

[16] D. Mumford, J. Shah, Optimal approximations by piecewise smooths functions and associated variational problems, Commun. Pure Appl. Math, Vol.42, no.5, (1989) 577-685.

[17] M. Tello Alonso, C. Lopez-Martinez and J.J. Mallorqui, P. Salembier, "edge enhancement algorithm based on the wavelet transform for automatic edge detection in SAR Images", IEEE transaction on geodesic and remote sensing, vol. 49, no.1, Jan 2011.

[18] T.E. Chan and L.A. Vese,"A level set algorithm for minimizing the mumford-shah functional in image processing", IEEE Workshop on Variational and Level Set Methods in Computer Vision, pp.161-168.

[19] C. Li, C. Kao, J. Gore, Z. Ding, "Implicit active contours driven by local binary fitting energy", IEEE Conference on Computer Vision and Pattern Recognition (CVPR), IEEE Computer Society, Washington, DC, USA, 2007, pp. 1-7.

[20] L. Vese, T. Chan, "A multiphase level set framework for image segmentation using the Mumford and Shah model", Int. J. Comput. Vis. 50 (3) (2002) 271-293. 
[21] C. Li, "Active contours with local binary fitting energy", IMA Workshop on New Mathematics and Algorithms for 3-D Image Analysis, 2006.

[22] C. Li, C. Xu, C. Gui, M.D. Fox, "Level set evolution without re-initialization a new variational formulation", IEEE Conference on Computer Vision and Pattern Recognition (CVPR), vol. 1, 2005, pp. 430-436.

\section{BIOGRAPHIES}

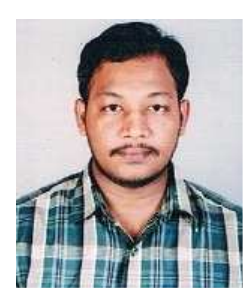

Ch. SriVeeraSagar received B.Tech degree in Electrical and Electronics Engineering. $\mathrm{He}$ is currently pursuing M.Tech degree in instrumentation and control systems from University College of Engineering, JNTU Kakinada. His area of interest is image processing in typical noised images.

Presently working on speckle noised images to detect the significant and delicate edges with the study of some of the existing works in different application and simulated in MatLab environment.

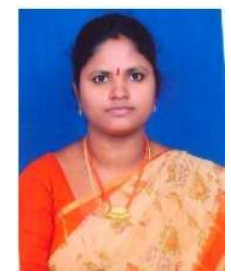

K. RamaDevi obtained M.E degree in Microwave and Radar Engineering from Osmania University College of Engineering in 2003. She is presently working as Assistant Professor in the Department of Electronics and Communication Engineering. She is presently pursuing Doctoral degree from JNTUK.

She presented many research papers in various national and international journals and conferences. Her research interests include Micro Strip Patch Antennas, slot antennas and image processing in radar applications simulated in MATLAB and HFSS. 